\title{
Impact of various extents of experimental subarachnoid hemorrhage induced by the endovascular filament model on mortality and changes of cerebral blood flow
}

\author{
Thomas Westermaier, MD*; Alina Jauss, MD; Giles H. Vince, MD; Furat Raslan, MD; Joerg Eriskat, MD; \\ Klaus Roosen, MD, PhD \\ Department of Neurosurgery, University of Wuerzburg, Wuerzburg, Germany
}

\begin{abstract}
Objective: Among various animal models of subarachnoid hemorrhage (SAH), the endovascular filament model has been found particularly suitable to investigate acute pathophysiological changes after experimental SAH. Its major drawback, however, are high mortality and high variability of results. The present studies were carried out to examine the impact of various extents of experimental SAH in rats induced by different filament sizes.

Methods: In study 1, Sprague-Dawley rats were subjected to vessel perforation with a 3-0, 4-0 or 5-0 monofilament or served as controls $(n=8)$. Intracranial pressure (ICP), mean arterial blood pressure (MABP), cerebral perfusion pressure (CPP) and local cortical blood flow (LCBF) were continuously monitored for 6 hours after $\mathrm{SAH} .24$ hours later, the animals were sacrificed to evaluate subarachnoid blood effusion. In study 2 , the animals were subjected to SAH using a 3-0 monofilament or served as controls $(n=8)$. After 7 days, they were sacrificed for quantification of tissue damage.

Results: With increasing filament size, the decrease of CPP and LCBF and subarachnoid blood effusion were more pronounced. In all SAH-groups, the decline of LCBF after SAH exceeded the decline of CPP. Hippocampal damage was moderate but consistent. In both studies, the acute mortality was lower than previously reported.

Conclusion: With controlled pre-hemorrhage blood pressure values and longer mechanical ventilation mortality can be kept low even if stronger filaments are used. The impact of subarachnoid vessel perforation on pathophysiological changes and blood effusion can be graded by the use of different filament sizes. The persisting mismatch between CPP and LCBF both in major and minor SAH suggests sustained vasoconstriction occurring independent of the extent of hemorrhage.
\end{abstract}

Keywords: brain subarachnoid hemorrhage; mortality; endovascular filament model; cerebral blood flow, model

\section{Introduction}

A variety of experimental models has been used in the last decades to examine pathophysiological changes after SAH. Recently, Lee et al. published a thorough comparison of the most frequently used experimental models of $\mathrm{SAH}$, the endovascular filament model and the double injection model (Lee et al, 2009). Similar to previous reports (Gules et al, 2002; Prunell et al, 2003), the authors found that the endovascular filament model (Bederson et al, 1995; Veelken et al, 1995) produced a more pronounced subarachnoid blood effusion, elevation of ICP and decrease of cerebral blood flow (CBF). Therefore, the endovascular filament model seems more suitable for the examination of the acute stage after SAH. In addition, it allows continuous monitoring of hemodynamic parameters and cerebral pathophysiological changes without violation of the intracranial vault and without repositioning of the animals or monitoringprobes (Critchley and Bell, 2003; Marshman, 2002; Schwartz et al, 2000; Sehba et al, 1999; Busch et al, 1998; Bederson et al, 1998). However, the model has been met with criticism. Most authors use a 3-0 nylon monofilament for vessel perforation in order to simulate high-grade $\mathrm{SAH}$ with a significant amount of subarachnoid blood and pronounced pathophysiological changes. High mortality rates during the acute stage of the experiment were, thus, reported. High mortality rates, in turn, make reliable conclusions difficult, in particular if a study focuses on mechanisms of secondary brain damage and neuroprotection (Lee et al, 2009).

Schwartz et al. reported that the intensity of SAH might be varied by the use of different filament sizes (Schwartz et al, 2000). It was the aim of the present study to characterize mortality and time-course of pathophysiological changes in the acute phase after experimental SAH using different filament sizes.

From the clinical point of view, the course of pathophysiological changes several hours after SAH is of particular interest since the majority of SAH-patients are admitted to hospitals in this range of time and secondary ischemic events might arise in this particu-

\section{* Correspondence should be sent to:}

Thomas Westermaier, Department of Neurosurgery, University of Wuerzburg, Josef-Schneider-Str. 11, 97080 Wuerzburg, Germany; Phone:+49 - 931- 24527; Fax:+49 - 931- 24157; E-mail: westermaier.t@nch.uni-wuerzburg.de 
lar period (Miyazaki et al, 2006; Sato et al, 2006; Audebert et al, 2005). However, this period is poorly characterized. Clinical observations using devices of neuromonitoring usually do not start before the obliteration of the aneurysm (Sarrafzadeh et al, 2005). However, the observation time in previous experimental studies has not been long enough to cover this particular time-span (Prunell et al, 2003; Schwartz et al, 2000; Bederson et al, 1998; Jackowski et al, 1990; Lee et al, 2009). Therefore, we extended the monitoring time to 6 hours after induction of $\mathrm{SAH}$.

\section{Materials and Methods}

For study 1, 36 male Sprague-Dawley rats (250-300 g body weight) were used. For study 2, 16 rats were used. All animals were purchased from Harlan Winkelmann (Borchen, Germany). All experiments were approved by the regional authorities and the district government of Bavaria, Germany.

\section{Animal Preparation and Monitoring}

The animals were anesthetized with $4 \%$ Isoflurane, orally intubated and mechanically ventilated with an air/oxygen mixture to maintain normal arterial blood gases. After induction of anesthesia, isoflurane was reduced to $2.5 \%$ for surgical procedures and to $2 \%$ from 30 minutes prior to SAH until the end of the monitoring period. Temporalis muscle and rectal probes were used to monitor the temperature throughout the experiment. For this purpose, a 21 gauge butterfly cannula was equipped with an industrial temperature sensor (Philips-Thermocoax, Stapelfeld, Germany) at its tip. It was inserted into the temporalis muscle and connected to a heating relais. A thermostatically regulated, feedback-controlled infrared heating lamp was positioned at a distance of 50 $\mathrm{cm}$ over the animal in order to maintain the temporalis muscle and rectal temperature at $37^{\circ} \mathrm{C}$. The tail artery was cannulated for continuous measurement of mean arterial blood pressure (MABP) and for blood sampling. Arterial blood gases were measured 30 minutes and 5 minutes before induction of $\mathrm{SAH}$ and in hourly intervals thereafter.

\section{Laser Doppler Flowmetry and ICP Measurement}

A two-channel laser Doppler flowmeter (LDF) (MBF3D; Moor Instruments, Axminster, England) was used for continuous monitoring of local cortical blood flow (LCBF) in the area of the cerebral cortex supplied by the MCA. In study 1, bilateral LCBF was measured, in study 2 only contralateral LCBF was measured. To place LDF probes, burr holes were drilled $5 \mathrm{~mm}$ lateral and $1 \mathrm{~mm}$ posterior to the bregma without injury to the dura mater. For ICP measurement, an additional burr hole was drilled over the right frontal cortex.
The animals were then placed in a supine position with the head fixed in a stereotactic frame with earbars. A rectangularly bent laser-Doppler probe was positioned in each burr hole with a micromanipulator. An intraparenchymal Camino ICP-probe (Integra Neurosciences, Plainsboro, NJ, USA) was advanced $2 \mathrm{~mm}$ into the brain by a third micromanipulator.

\section{Induction of $\mathrm{SAH}$}

SAH was induced by use of the endovascular puncture method (Veelken et al, 1995; Bederson et al, 1995). After surgical exposure of the right cervical carotid bifurcation, temporary aneurysm clips were placed on the common and internal carotid artery. A Prolene ${ }^{\odot}$ filament (Ethicon, Inc., Somerville, NJ) was inserted into the external carotid artery and fixed with a silk ligature and the temporary clips were removed. After a stabilization period of 30 minutes, the filament was advanced into the internal carotid artery (ICA) until a resistance was felt. The filament then was pushed 2-3 mm further for intracranial vessel perforation. The suture was then quickly withdrawn into to allow reperfusion and development of SAH. SAH was confirmed by a rapid decrease of LDF and increase of ICP.

\section{Experimental groups}

Study 1: The rats were randomly assigned to one of four groups ( $n=8$ for each group): 1 ) Perforation with a 3-0 Prolene filament, 2) perforation with a 4-0 Prolene filament, 3) perforation with a 5-0 Prolene filament and 4) sham operated rats. In the latter group, a 3-0 filament was inserted into the ICA without vessel perforation.

Study 2: The animals were randomly assigned to one of two groups ( $n=8$ for each group): 1 ) Perforation with a 3-0 filament, 2) sham-operation.

Termination of operative procedures and wound closure

Six hours after induction of $\mathrm{SAH}$, monitoring was stopped, the ICP probe and laser Doppler probes and the arterial catheter were removed and the wounds were closed with a skin suture. Isoflurane was withdrawn and the animals were allowed to wake up.

Quantification of neurological performance and amount of subarachnoid blood

In animals of study 1 , neurological performance was assessed 30 minutes after withdrawal of isoflurane by an examiner blinded to the animals' study group. Activity was assessed using a 5 point grading scale: 4) normal spontaneous activity, 3) slightly reduced spontaneous activity, 2) activity following manipulation 1) activity only following painful stimulus, 0) animal dead. 24 hours later, neurological evaluation was repeated and the animals were euthanized. The - 9 - 
brains removed and the amount of subarachnoid blood was quantified using a semiquantitative scale: 0) No blood visible; 1 ) traces of blood visible; 2 ) unilateral clot; 3) generalized bilateral basal blood clot; 4) intracerebral hematoma with or without subarachnoid blood.

In animals of study 2, neurological examination was performed using the respective tests 30 minutes after termination of operative procedures and each day thereafter for seven days. At day 7 after SAH, the animals were anesthetized and transcardially perfused with $2 \%$ paraformaldehyde. The brains were removed, embedded in paraffin and cut into 4- $\mu \mathrm{m}$ thick coronal sections at $400-\mu \mathrm{m}$ intervals, which were stained with cresyl violet. Three defined parts of the CA1 region of the hippocampus (bregma -3.24, 4.92, -6.12) were determined according to a stereotactic atlas of the rat brain (Paxinos $\mathrm{G}$ and Watson $\mathrm{C}$, 2005), bilaterally analyzed for surviving neurons, and their number per field $(0.2 \times 0.3 \mathrm{~mm})$ was counted.

\section{Statistical analysis}

Statistical analysis was performed with SPSS 14.0 (SPSS, Inc., Chicago, IL). Physiological data for each time point, LDF and ICP data were analyzed with a one-way analysis of variance (ANOVA). When multiple comparisons were indicated, a Bonferroni correction was applied. $\mathrm{P}<0.05$ was considered significant. Results are presented as mean \pm standard deviation (SD).

\section{Results}

\section{Study 1}

Two animals had to be excluded due to misplacement of the endotracheal tube. Two further animals in the 5-0 group were excluded from the study and replaced because advancing the filament did not result in characteristic changes of ICP and LCBF.

\section{Physiological parameters}

Prior to $\mathrm{SAH}$, the mean values of $\mathrm{pH}$ and the partial pressures of carbon oxide $\left(\mathrm{pCO}_{2}\right)$ and oxygen $\left(\mathrm{pO}_{2}\right)$ in arterial blood samples were $7.408 \pm 0.12,40.73 \pm$ 9 and $139.1 \pm 28$, respectively. After SAH, respirator settings were adjusted according to hourly blood gas analyses to keep arterial blood gases within the normal range. There were no significant differences between the groups regarding $\mathrm{pH}, \mathrm{pCO}_{2}$ and $\mathrm{pO}_{2}$.

\section{ICP, MABP, CPP and LCBF}

The courses of ICP, MABP and CPP from 30 minutes before until 6 hours after induction of SAH are depicted in Fig. 1a-c. The course of ipsi- and contralateral LCBF is depicted in Fig. $2 \mathrm{a}$ and $\mathrm{b}$.

\section{Extent of hemorrhage}

After perfusion fixation and removal of the brain, no traces of blood were found in the subarachnoid space of the brains of control animals. In the 3-0 group, two animals had extensive SAH in the basal subarachnoid space and a small intracerebral hematoma and subdural hematoma over the frontobasal surface on the right side. In two animals blood was spread over the entire basal subarachnoid space without intracerebral/subdural hematoma. In the remaining 4 animals a unilateral subarachnoid blood clot was found. In the 4-0 group, one animal had the entire basal subarachnoid space filled with blood, four had a localized blood clot, 3 only minor traces of blood. In the 50 group, 5 animals had a localized clot, three animals only minor traces of blood (Fig. 3).

\section{Mortality and neurological performance}

In the control group, all animals showed normal activity after termination of anesthesia and 24 hours later. In the 3-0 group, 2 animals did not regain consciousness, had a pathological respiration pattern after termination of anesthesia and were euthanized shortly thereafter. Those were the two animals with intracerebral and subdural hematoma. After 24 hours, one further animal with extensive basal SAH showed activity only after minor manipulation, one showed activity only after painful stimuli. Two animals showed slightly reduced spontaneous activity, two showed normal activity. In the 4-0 and in the 5-0 group, all animals woke up regularly after the end of the observation period and cessation of anesthesia. In the 4-0 group one animal deteriorated and had to be euthanized earlier than 24 hours after SAH. This animal had extensive basal SAH without intracerebral/subdural hematoma. In the 5-0 group one animal deteriorated and had to be euthanized earlier than 24 hours after SAH. This animal had a unilateral blood clot (Fig. 4).

\section{Study 2}

\section{Physiological parameters:}

There were no significant differences between the two groups regarding $\mathrm{pH}, \mathrm{pCO}_{2}$ and $\mathrm{pO}_{2}$. Intracranial pressure, arterial blood pressure, cere-
bral perfusion pressure

In the SAH-group, ICP increased from a baseline of 8 $\pm 4 \mathrm{mmHg}$ to a maximum of $64 \pm 36 \mathrm{mmHg}$ five minutes after $\mathrm{SAH}$, declined to $18 \pm 13 \mathrm{mmHg}$ after 1 hour and to $15 \pm 11 \mathrm{mmHg}$ at the end of the observation period. In the control group, baseline ICP was 8 $\pm 5 \mathrm{mmHg}$ and showed no relevant changes throughout the monitoring time. The increase of ICP was significant throughout the whole observation time. MABP increased from a baseline of $82 \pm 7 \mathrm{mmHg}$ to a maximum of $116 \pm 46 \mathrm{mmHg}$ and declined to $84 \pm$ $17 \mathrm{mmHg}$ one hour after SAH and $78 \pm 17 \mathrm{mmHg}$ -10 - 
after 6 hours. Baseline MABP in the control group was $82 \pm 10 \mathrm{mmHg}$ and remained stable to reach 87 $\pm 18 \mathrm{mmHg}$ after 6 hours. The increase of MABP was significant 5 minutes after SAH. CPP declined from $74 \pm 10 \mathrm{mmHg}$ to a minimum of $41 \pm 27 \mathrm{mmHg} 1$ minute after $\mathrm{SAH}$ and recovered to $66 \pm 14 \mathrm{mmHg}$ one hour after SAH and remained stable around 70 $\mathrm{mmHg}$ until the end of observation period. Baseline CPP in the control group was $74 \pm 12 \mathrm{mmHg}$ and increased slightly to reach an endpoint of $80 \pm 18$ $\mathrm{mmHg}$ after 6 hours. The decline of CPP was significant until 5 minutes after SAH.

\section{Local Cerebral Blood Flow}

LCBF declined to a minimum of $20 \pm 11 \%$ of baseline one minute after SAH and recovered to $54 \pm 20 \%$ after 60 minutes and increased further to $73 \pm 30 \%$ at the end of the observation period. In the control group LCBF remained stable throughout the observation period to reach $102 \pm 33 \%$ after 6 hours. The decrease of LCBF was significant compared to the control group from the onset of SAH until 240 minutes thereafter and 330 minutes after $\mathrm{SAH}$.
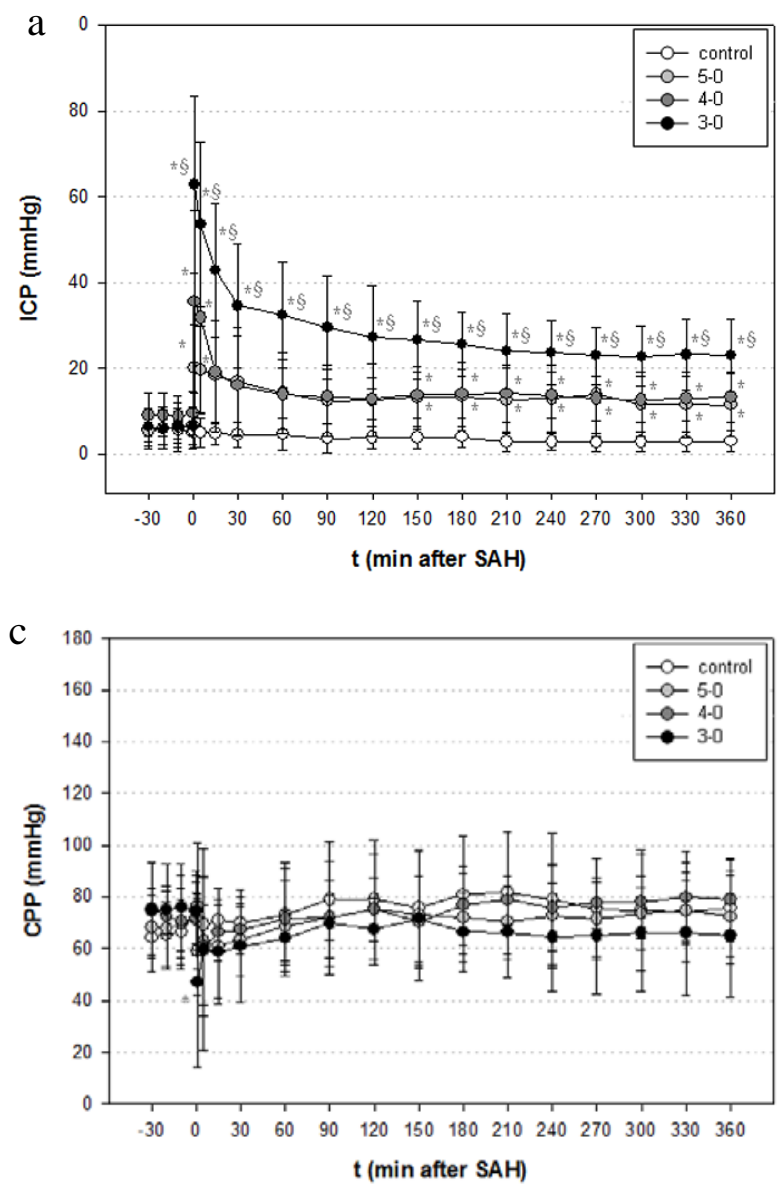

\section{Mortality and neurological performance}

No animal had to be excluded from the study. In the control group, there was no operative mortality and all animals showed normal activity after operative procedures and in the 7 days thereafter. In the SAHgroup, there was no mortality during monitoring time or during the following 24 hours. One animal had to be sacrificed on day 2 after $\mathrm{SAH}$, another animal on day 3 because of secondary deterioration. The other animals recovered well from operative procedures and $\mathrm{SAH}$ and showed normal activity or slightly reduced activity.

\section{Histological damage}

In the control group, $59 \pm 15$ CA-1 neurons per visual field were counted in the right, $60 \pm 15$ in the left hippocampus. In the SAH-group, $53 \pm 10 \mathrm{CA}-1$ neurons were counted in the right, $53 \pm 8$ in the left hippocampus. No territorial or lacunar infarctions were visible in either hemisphere as a sign of vessel occlusion or cerebral vasospasm.

b

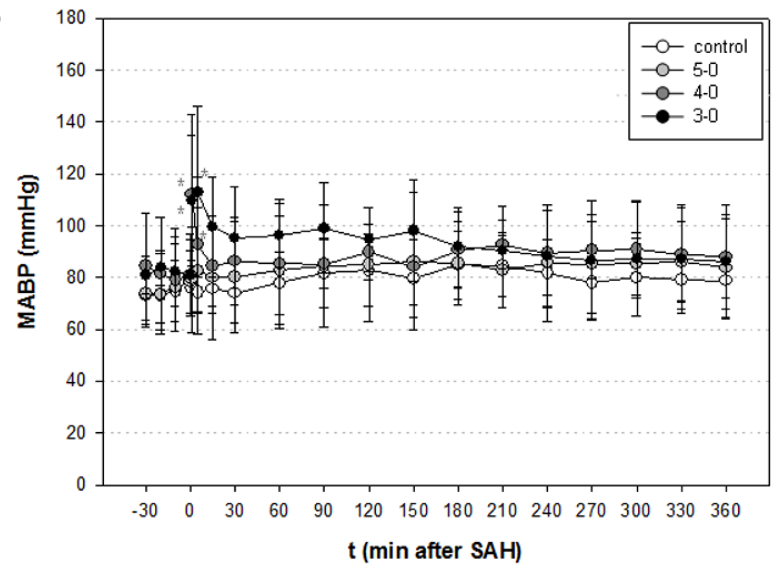

Fig. 1a-c: Time course of intracranial pressure (1a), mean arterial blood pressure (1b) and cerebral perfusion pressure (1c) monitored from 30 minutes before until 6 hours after SAH induced by vessel perforation by an intraluminal filament. ICP reached a nadir approximately two minutes after $\mathrm{SAH}$. Secondary to the rapid elevation of ICP, arterial blood pressure rose in the 3-0 and 4-0 groups. Thus, CPP was only significantly elevated one minute after SAH in the 3-0 group. $\left({ }^{*} \mathrm{p}<0.05\right.$ vs. control group, $\$ p<0.05$ vs. $4-0$ and $5-0$ groups) 

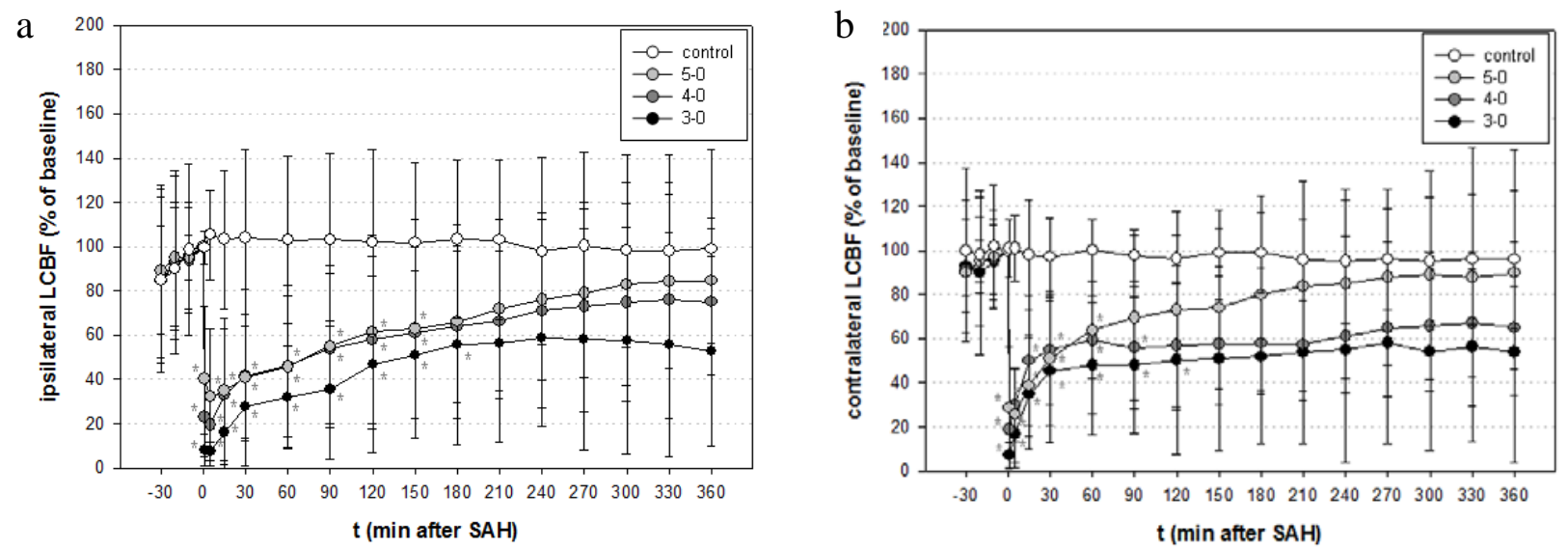

Fig. 2 a and b: Local cortical Blood Flow (LCBF) measured by continuous laser-Doppler flowmetry over the ipsilateral (2a) and contralateral (2b) hemisphere from 30 minutes before until 6 hours after induction of SAH. The decrease of LCBF and its recovery varies with the size of the filament used for vessel perforation with more pronounced decrease and slower recovery when stronger filaments are used. However, a mismatch between the only little and insignificant reduction of CPP (see Fig. 1c) and the marked and significant reduction of LCBF was found in all three SAH groups indicating acute vasoconstriction even with smaller filament sizes. The mismatch between CPP- and LCBF-reduction started at the onset of SAH and persisted until the end of the observation period. ( ${ }^{*} p<0.05$ vs. control group)

Extent of subarachnoid blood

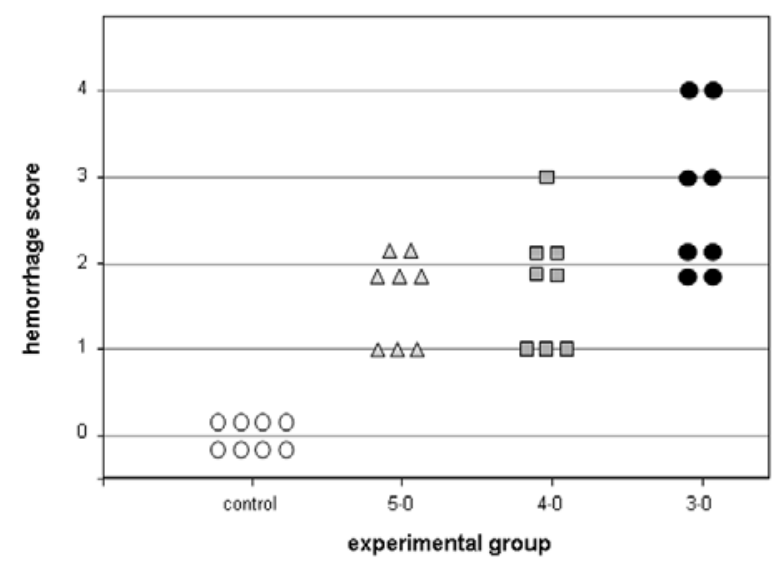

Fig. 3: Amount of subarachnoid blood determined by a semiquantitative visual score analogous to the Fisher score: 0) No blood visible; 1) traces of blood visible, no blood clot: 2) unilateral clot; 3) generalized bilateral basal blood clot; 4) intracerebral hematoma with or without subarachnoid blood.

\section{Discussion}

\section{Mortality}

The present experiments were performed to characterize the impact of SAH induced by the endovascular filament model of SAH on ICP, changes of brain
Activity 24 hours after SAH

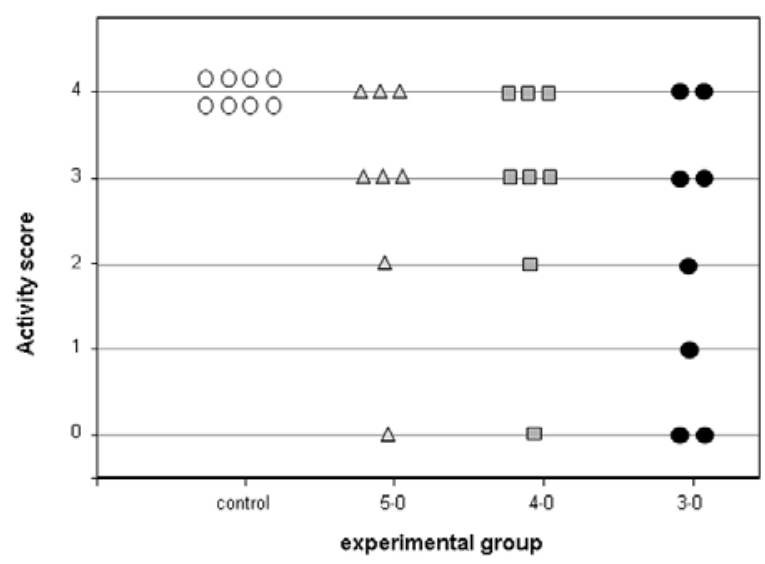

Fig. 4: Graph depicting assessment of activity 24 hours after anesthesia was stopped. A 5 point grading scale was used as follows: 4) normal spontaneous activity, 3) slightly reduced spontaneous activity, 2) no spontaneous activity, reaction on painful stimulus, 1) no activity on stimulus, 0) animal dead.

perfusion and mortality using different filament sizes. The endovascular filament model of SAH has first been described in 1995 by Bederson et al. and Veelken et al (Bederson et al, 1995; Veelken et al, 1995). Compared to other experimental models it has certain advantages: 1) the skull and arachnoid are not 
opened by a craniotomy or cisternal puncture, 2) the origin of hemorrhage is located in the basal subarachnoid space similar to aneurysmal $\mathrm{SAH}$ and 3 ) by perforation of a blood vessel potentially vasoactive components of the vessel's endothelial or muscular layer are exposed to the subarachnoid space similar to an aneurysm rupture. These features make the endovascular filament model particularly suitable for the investigation of the acute phase of SAH (Lee et al, 2009). However, it has also been met with criticism because of high mortality rates of $40-100 \%$ (Veelken et al, 1995; Bederson et al, 1995; Gules et al, 2002; Prunell et al, 2003; Lee et al, 2009) and higher standard deviations of results (Prunell et al, 2003; Prunell et al, 2004). In all studies cited above, a 3-0 nylon monofilament was used for vessel perforation in rats and $\mathrm{SAH}$ was induced following the description of Bederson et al. (Bederson et al, 1995). We proceeded likewise, however, could neither reproduce these high mortality rates if a smaller filament was used nor if a 3-0 monofilament was used for vessel perforation. Comparing our data to the experimental setup and the results of previously published studies, we find differences with regard to prehemorrhage blood pressure values and the conduction and duration of anesthesia. The period of continuous monitoring of ICP, CPP and regional CBF in our experiments was chosen to be 360 minutes after $\mathrm{SAH}$. The primary aim of the extended monitoring time was to obtain information about the long-term course of pathophysiological changes. But, naturally, the extended monitoring time also required anesthesia and mechanical ventilation for 360 minutes after SAH.

In a previous study, Bederson and coworkers found that a reduction of CBF to less than $40 \%$ of baseline for 60 minutes after SAH were linked to $100 \%$ mortality. In that study, mean ICP-values in the group of non-survivors after 60 minutes were $30 \mathrm{mmHg}$. (Bederson et al, 1995). In our experiments, mean values of ICP and LCBF in the 3-0 group were comparable to the study of Bederson et al. but mortality was much lower. In study 1, two animals of the 3-0 group had to be sacrificed after the acute stage of the experiment because of poor neurological performance and a pathological respiration pattern, clinical features indicating elevated ICP. In fact, those two animals had acute subdural hematoma and were the ones within the 3-0 group with the lowest LCBF and highest ICP after 6 hours (38 and $30 \mathrm{mmHg}$ ). Following an initial ICP-peak after induction of $\mathrm{SAH}$, these animals, at first, showed regular recovery of ICP and LCBF in the first minutes after hemorrhage. However, between 2 and 6 hours after SAH, no further significant improvement occurred. In the other animals of the 3-0 group and in the (3-0) SAH-group of study 2, further continuous recovery was observed within 2 and 6 hours after $\mathrm{SAH}$.

These observations indicate that early termination of anesthesia and mechanical ventilation might leave the animals with ICP-values that might be too high to guarantee appropriate spontaneous ventilation and other vital functions. Longer mechanical ventilation and airway protection may, therefore, have helped the animals overcome the first critical hours until ICP and LCBF returned to tolerable values. Although high mortality rates might represent the clinical situation after aneurysmal SAH, they might distort experimental results, in particular, because those animals are not eligible for neurological assessment and histological analysis. The advantages of an experimental model like consistency of the experimental setup and lack of selection bias may, therefore, be annihilated.

\section{Tissue damage}

Assuming that a bilateral decrease of laser-Doppler flow (LDF) reflects a global reduction of CBF, that baseline LDF reflects normal cerebral perfusion and that relative changes of LDF reflect relative changes of CBF in about the same percentage (Soehle et al, 2000; Schmid-Elsaesser et al, 1998), CBF did not fall below ischemic thresholds in any of the SAH-groups (Jones et al, 1981). Accordingly, we found no signs of cerebral infarction. In contrast, we observed minor, but rather consistent hippocampal damage suggesting a damage due to chronic hypoperfusion which is in accordance with other reports (Lee et al, 2009; Westermaier et al, 2009; Prunell et al, 2003). With respect to the low mortality-rates observed in the present experiments, even neuroprotection-studies might be considered using the endovascular filament model.

\section{Acute Vasoconstriction}

In previous experiments (Westermaier et al, 2009), we observed a mismatch of the recovery of CPP and the recovery of LCBF after SAH. This is in analogy to the reports of other groups (Bederson et al, 1998; Schwartz et al, 2000; Lee et al, 2009) and suggests direct vasoconstriction. The present data furthermore demonstrates that this acute-type vasoconstriction occurs in experimental subarachnoid hemorrhage of various - even minor - extent and may persist for hours after cerebral perfusion pressure has already recovered.

After induction of $\mathrm{SAH}$, only a minor and very shortlasting decrease of CPP was observed. LCBF, in contrast, was markedly reduced. LCBF partially recovered with better and faster recovery when smaller filaments were used for vessel perforation. However, a discrepancy between CPP and LCBF was observed in all three SAH-groups starting immediately after SAH and persisting throughout the entire 6-hour -13 -

J Exp Stroke Transl Med (2011) 4(1): 8-15

Society for Experimental Stroke (www.s4es.org) 
observation period. While LCBF well recovered in the 5-0 group, it remained markedly below baseline levels in the 3-0 and 4-0 groups. This finding indicates that an immediate and uniform vascular reaction occurs in the in the first hours after SAH independent of the extent of hemorrhage. Long-term recovery, in turn, seems to be influenced by the extent of hemorrhage as LCBF showed better recovery when smaller filament sizes were used.

Previous studies using this model examined the first one to three hours after induction of SAH (Bederson et al, 1998; Schwartz et al, 2000; Prunell et al, 2003; Jackowski et al, 1990). In clinical practice, however, the diagnosis of aneurysmal SAH in the majority of cases is confirmed not earlier than 3 to 6 hours after aneurysm rupture (Miyazaki et al, 2006; Sato et al, 2006; Audebert et al, 2005). For this particular period, there is a lack of information. A biphasic vascular reaction after $\mathrm{SAH}$ with an acute and a delayed vasospasm has been reported by several authors (Peerless et al, 1982; Delgado et al, 1985; Svendgaard et al, 1985). Under controlled experimental conditions, Bederson et al. characterized the early phase of vasoconstriction by continuous monitoring of pathophysiological changes for 60 minutes after $\mathrm{SAH}$ using the same experimental setup (Bederson et al, 1998). To cover this time-span, hemodynamic and CBF-monitoring was extended for 6 hours after the induction of $\mathrm{SAH}$. Our experiments demonstrate that acute vasoconstriction may be an enduring phenomenon which lasts for several hours after SAH and even occurs in minor $\mathrm{SAH}$.

\section{Conclusion}

The high mortality rates reported by other authors could not be reproduced in the present experiments. The most important reason most likely is longer mechanical ventilation and airway protection. By this way, a selection bias might be reduced as survive that would otherwise have to be excluded from neurological and histological evaluation. Hence, we found moderate, but consistent hippocampal damage and even neuroprotection studies might be performed with this experimental model.

If pre-SAH setup is constant, however, the impact of experimental SAH induced by the endovascular filament model can be graded by the use of different filament sizes. The mismatch between decreases of CPP and LCBF over both hemispheres in all experimental groups indicates the occurrence of a generalized acute vasoconstriction starting immediately after induction of SAH and persisting for at least 6 hours. This mismatch occurred independent of the extent of $\mathrm{SAH}$. From the present results, the origin and exact location within the cerebral vascular system of this phenomenon cannot be determined. Additional experiments like continuous visualization of cerebral vessels have to be performed.

\section{Acknowledgement}

None

\section{Conflict of interest}

None

\section{References}

Audebert HJ, Clarmann VC, Schenkel J, Furst A, Ziemus B, Metz C, Haberl RL. (2005) Problems of emergency transfers of patients after a stroke. Results of a telemedicine pilot project for integrated stroke accommodation in southeast Bavaria (TEMPiS). Dtsch Med Wochenschr 130:2495-2500

Bederson JB, Germano IM, Guarino L. (1995) Cortical blood flow and cerebral perfusion pressure in a new noncraniotomy model of subarachnoid hemorrhage in the rat. Stroke 26:1086-1091

Bederson JB, Levy AL, Ding WH, Kahn R, DiPerna CA, Jenkins AL, Vallabhajosyula P. (1998) Acute vasoconstriction after subarachnoid hemorrhage. Neurosurgery 42:352-360

Busch E, Beaulieu C, de Crespigny A, Moseley ME. (1998) Diffusion MR imaging during acute subarachnoid hemorrhage in rats. Stroke 29:2155-2161

Critchley GR, Bell BA. (2003) Acute cerebral tissue oxygenation changes following experimental subarachnoid hemorrhage. Neurol Res 25:451-456

Delgado TJ, Brismar J, Svendgaard NA. (1985) Subarachnoid haemorrhage in the rat: angiography and fluorescence microscopy of the major cerebral arteries. Stroke 16:595-602

Gules I, Satoh M, Clower BR, Nanda A, Zhang JH. (2002) Comparison of three rat models of cerebral vasospasm. Am J Physiol Heart Circ Physiol 283:2551-2559

Jackowski A, Crockard A, Burnstock G, Russell RR, Kristek F. (1990) The time course of intracranial pathophysiological changes following experimental subarachnoid haemorrhage in the rat. $\mathrm{J}$ Cereb Blood Flow Metab 10:835-849

Jones TH, Morawetz RB, Crowell RM, Marcoux FW, FitzGibbon SJ, DeGirolami U, Ojemann RG. (1981) Thresholds of focal cerebral ischemia in awake monkeys. $\mathrm{J}$ Neurosurg 54:773-782

Lee JY, Sagher O, Keep R, Hua Y, Xi G. (2009) Comparison of experimental rat models of early brain injury after subarachnoid hemorrhage. Neurosurgery 65:331343

Marshman LA. (2002) Preserved contractility without side bias in endovascular filament models for subarachnoid hemorrhage. J Neurosci Methods 117:193-200 
Miyazaki T, Ohta F, Moritake K, Nagase A, Kagawa T. (2006) The key to improving prognosis for aneurysmal subarachnoid hemorrhage remains in the prehospitalization period. Surg Neurol 65:360-5

Paxinos G, Watson C. ( 2005) The Rat Brain in stereotaxic Coordinates. 5th Edn. San Diego, Elsevier Academic Press.

Peerless SJ, Fox AJ, Komatsu K, Hunter IG. (1982) Angiographic study of vasospasm following subarachnoid hemorrhage in monkeys. Stroke 13:473-479

Prunell GF, Mathiesen T, Diemer NH, Svendgaard NA. (2003) Experimental subarachnoid hemorrhage: subarachnoid blood volume, mortality rate, neuronal death, cerebral blood flow, and perfusion pressure in three different rat models. Neurosurgery 52:165-175

Prunell GF, Mathiesen T, Svendgaard NA. (2004) Experimental subarachnoid hemorrhage: cerebral blood flow and brain metabolism during the acute phase in three different models in the rat. Neurosurgery 54:426-436

Sarrafzadeh A, Haux D, Plotkin M, Ludemann L, Amthauer $\mathrm{H}$, Unterberg A. (2005) Bedside microdialysis reflects dysfunction of cerebral energy metabolism in patients with aneurysmal subarachnoid hemorrhage as confirmed by $15 \mathrm{O}-\mathrm{H} 2 \mathrm{O}-\mathrm{PET}$ and 18 F-FDG-PET. J Neuroradiol 32:348-351

Sato M, Nakano M, Asari J, Watanabe K. (2006) Admission blood glucose levels and early change of neurological grade in poor-grade patients with aneurysmal subarachnoid haemorrhage. Acta Neurochir (Wien) 148:623-626

Schmid-Elsaesser R, Zausinger $S$, Hungerhuber $E$, Baethmann A, Reulen HJ. (1998) A critical reevalua- tion of the intraluminal thread model of focal cerebral ischemia: evidence of inadvertent premature reperfusion and subarachnoid hemorrhage in rats by laserDoppler flowmetry. Stroke 29:2162-2170

Schwartz AY, Masago A, Sehba FA, Bederson JB. (2000) Experimental models of subarachnoid hemorrhage in the rat: a refinement of the endovascular filament model. J Neurosci Methods 96:161-167

Sehba FA, Ding WH, Chereshnev I, Bederson JB. (1999) Effects of S-nitrosoglutathione on acute vasoconstriction and glutamate release after subarachnoid hemorrhage. Stroke 30:1955-1961

Soehle M, Heimann A, Kempski O. (2000) Laser Doppler scanning: how many measurements are required to assess regional cerebral blood flow? Acta Neurochir Suppl 76:181-184

Svendgaard NA, Brismar J, Delgado TJ, Rosengren E. (1985) Subarachnoid haemorrhage in the rat: effect on the development of vasospasm of selective lesions of the catecholamine systems in the lower brain stem. Stroke 16:602-608

Veelken JA, Laing RJ, Jakubowski J. (1995) The Sheffield model of subarachnoid hemorrhage in rats. Stroke 26:1279-1283

Westermaier T, Jauss A, Eriskat J, Kunze E, Roosen K. (2009) Time-course of cerebral perfusion and tissue oxygenation in the first $6 \mathrm{~h}$ after experimental subarachnoid hemorrhage in rats. $\mathrm{J}$ Cereb Blood Flow Metab 29:771-779 